Article

\title{
Wear Behavior of Medium-Carbon Steel with Different Laser-Textured Densities under Starved Lubrication
}

\author{
Fanming Zeng ${ }^{1}$, Yuting Liu ${ }^{1}$, Feixian Shao ${ }^{2}$, Xiujuan $\mathrm{Li}^{2}{ }^{2}$, Zhenglei $\mathrm{Yu}^{2,3, *}$, Yunting Guo ${ }^{2}$, \\ Zhenping Wan ${ }^{4}$, Longsheng $\mathrm{Lu}^{4}{ }^{4}$ and Zhihui Zhang ${ }^{2,3}$ \\ 1 School of Material Science and Engineering, Changchun University of Science and Technology, \\ Changchun 130022, China; zengfm@126.com (F.Z.); yuting11029@163.com (Y.L.) \\ 2 The Key Laboratory of Bionic Engineering, Ministry of Education, Jilin University, Changchun 130022, China; \\ zzxu20@jlu.edu.cn (F.S.); xiujuanli@jlu.edu.cn (X.L.); yuntingguo93@163.com (Y.G.); zhzh@jlu.edu.cn (Z.Z.) \\ 3 State Key Laboratory of Automotive Simulation and Control, Jilin University, Changchun 130022, China \\ 4 School of Mechanical \& Automotive Engineering, South China University of Technology, \\ Guangzhou 510641, China; zhpwan@scut.edu.cn (Z.W.); meluls@scut.edu.cn (L.L.) \\ * Correspondence: zlyu@jlu.edu.cn
}

Received: 23 November 2020; Accepted: 10 December 2020; Published: 15 December 2020

\begin{abstract}
Five densities of laser-ablated micro-pits were fabricated onto medium-carbon steel surfaces based on the coupling effect of the biological surface. The effects of the surface textures on the wear behavior were investigated by sliding wear tests under starved lubrication. The results show that compared with the smooth sample, the biomimetic samples could effectively reduce friction and wear, and the tribological performance of the textured surfaces is closely related to the density of the bionic units. The equivalent stress distribution of the specimens was simulated by the finite element method. A uniform stress distribution is beneficial for effectively reducing the generation of wear cracks.
\end{abstract}

Keywords: medium-carbon steel; friction and wear behavior; biomimetic coupling surface; laser; starved lubrication

\section{Introduction}

The surfaces of factory equipment parts are susceptible to wear [1] and lubrication technology is often applied to improve the tribological performance of metal surfaces [2,3]. However, in the actual work environment, machines are often performing under starved-lubrication conditions [4-6]. Under these circumstances, the contact area of the friction pair cannot be filled with lubricating oil, as this will cause equipment failure and unnecessary economic loss [7-10]. In recent years, the tribological property of a friction pair in the case of starved lubrication has attracted widespread attention from researchers [11,12].

The proper modification of surface morphology is an important method used for enhancing the wear resistance of structural parts [13-15]. The micro-dimples not only serve as a micro-hydrodynamic bearing under a full- or mixed-lubrication regime [16,17], but also as a micro-reservoir for lubricant under starved-lubrication conditions. Additionally, the micro-dimples can also serve as a micro-trap for wear debris in either lubricated or dry sliding [18-22]. Two lubrication effects of surface texturing have been theoretically researched, namely, the hydrodynamic effect that mainly occurs under fullor mixed-lubrication conditions [23-25] and the secondary lubrication that occurs under mixedor starved-lubrication conditions [26]. We can see evidence in the natural world of wear-resistant properties of frictional surfaces due to the optimization of surface morphologies. For example, the earthworm, which scrapes against the surrounding soil and sandstone, has excellent wear-resistant 
mechanisms on its body surface. This wear-resistant mechanism is especially important when noting that the earthworm has a non-smooth morphology (such non-smooth morphologies, which are different from the microstructure of the substrate, are called biomimetic units) and exudes fluids [27] that form a protective film between its body surface and the soil.

In this study, the dimple-shaped and biomimetic units designed on the surface of medium-carbon steel were based on the non-smooth body surface and self-lubricating function of the earthworm. These units were fabricated by laser surface-texturing technology [28-30]. The effects of these units with different texture densities on the friction coefficient, wear-weight losses, and worn morphologies of the specimens were investigated under starved lubrication in order to obtain an optimized surface morphology, structure, and distribution to resist wear under starved lubrication. The experimental results and implications are discussed in this paper.

\section{Experimental Procedures}

\subsection{Specimens Preparation and Characterization}

The chemical compositions of medium-carbon steel and GCr15 are presented in Table 1. The specimens were cut to $25 \times 25 \times 10 \mathrm{~mm}^{3}$. The biomimetic surfaces of the specimens were manufactured by an Nd: YAG Laser (Directed Light Inc. San Jose, CA, USA) with a wavelength of $1.06 \mathrm{~mm}$, and the schematic diagram of sample preparation is shown in Figure 1. During the experiment, the spot diameter was $0.4 \mathrm{~mm}$, the laser output energy was $4 \mathrm{~J}$, and the maximum laser power was $800 \mathrm{~W}$. The prototype of the sample surface is the micro-pits covering the surface of the crucible. Table 2 shows the laser parameters for processing bionic units. The geometric parameters of the biomimetic samples are shown in Table 3, in which the density is the area ratio of the biomimetic surfaces to the total surface. The morphology of a single biomimetic unit is shown in Figure 2, which was observed by using a laser scanning confocal microscope (LSCM) (Olympus LEXT OLS 3000, Tokyo, Japan). The microstructures of the bionic units were analyzed by scanning electron microscopy (SEM) (Zeiss EVO 18 Special Edition, Jena, Germany). An X-ray diffractometer (XRD) (BRUKER D8 FOCUS X, karlsruhe, Germany) was used to analyze the phase of medium-carbon steel both treated by laser and untreated. The schematic arrangement of the laser texturing experiment is shown in Figure 1.

Table 1. The chemical constituents of medium-carbon steel and GCr15 were analyzed by EDS.

\begin{tabular}{cccccccccc}
\hline \multicolumn{2}{c}{ Elements } & C & Si & Mn & S & P & Cr & Mo & Ni \\
\hline $\begin{array}{c}\text { Composition } \\
\text { (wt.\%) }\end{array}$ & Medium-carbon steel & 0.45 & 0.21 & 0.54 & 0.002 & 0.015 & 0.057 & 0.002 & 0.016 \\
GCr15 & 0.98 & 0.18 & 0.37 & 0.003 & 0.02 & 1.55 & 0.038 & 0.016 \\
\hline
\end{tabular}

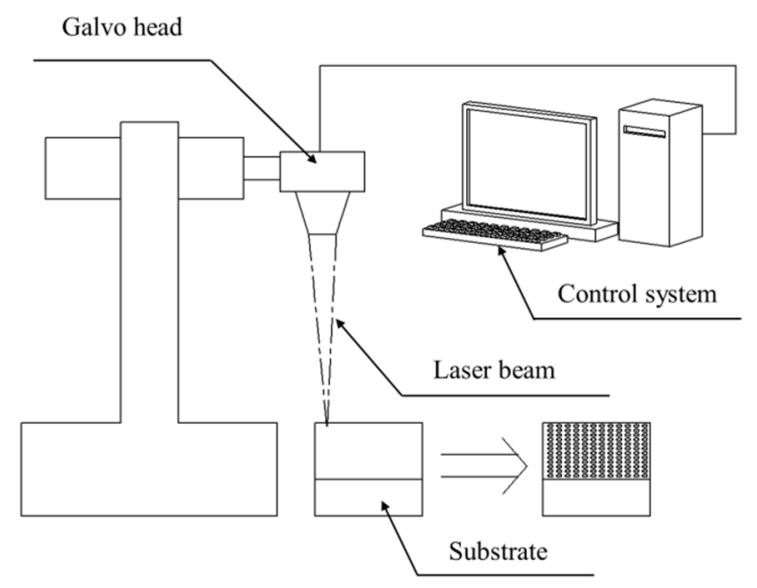

Figure 1. The experimental scheme of the laser-textured surface. 
Table 2. Processing parameters of the biomimetic samples.

\begin{tabular}{ccccc}
\hline Number & Electricity (A) & Pulse Duration $(\mathbf{m s})$ & Frequency $(\mathbf{H Z})$ & Scanning Speed $(\mathbf{m m} / \mathbf{s})$ \\
\hline 1 & 180 & 20 & 1 & 2.0 \\
2 & 180 & 20 & 1 & 2.5 \\
3 & 180 & 20 & 1 & 3.0 \\
4 & 180 & 20 & 1 & 3.5 \\
5 & 180 & 20 & 1 & 5.0 \\
\hline
\end{tabular}

Table 3. Geometric parameters of the biomimetic samples.

\begin{tabular}{ccccc}
\hline Number & Depth $(\boldsymbol{\mu m})$ & Diameter $(\boldsymbol{\mu m})$ & Center Distance $(\mathbf{m m})$ & Density of Dimples \\
\hline 1 & 120 & 1200 & 2.0 & $30 \%$ \\
2 & 120 & 1200 & 2.5 & $18 \%$ \\
3 & 120 & 1200 & 3.0 & $13 \%$ \\
4 & 120 & 1200 & 3.5 & $8 \%$ \\
5 & 120 & 1200 & 5.0 & $4 \%$ \\
\hline
\end{tabular}

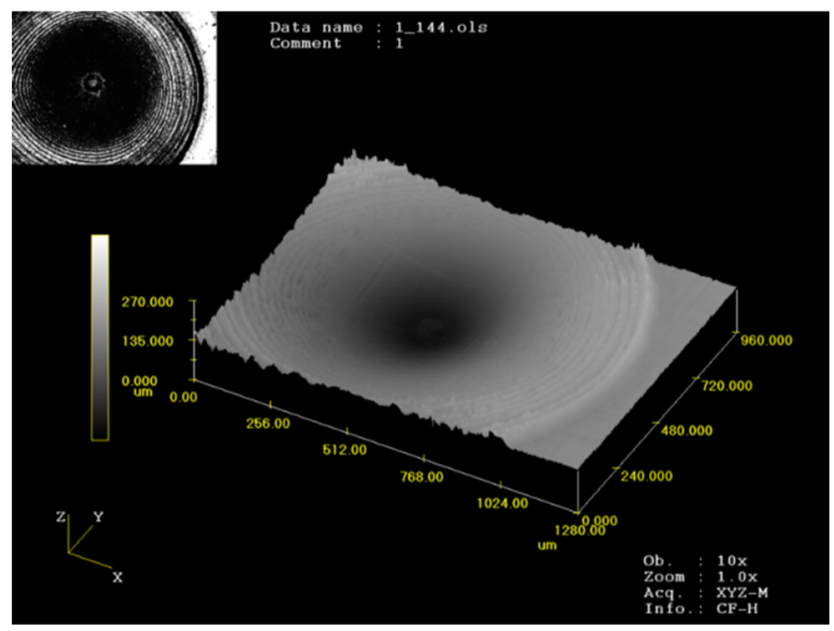

Figure 2. The stereoscopic profile of a single biomimetic unit.

\subsection{Friction and Wear Examination}

Sliding tests were carried out on an MG-2000 pin-on-disc at room temperature. The wear tests are shown in Figure 3. To study the effect of different working conditions on tribological properties, a series of experiments were designed, as shown in Table 4 . The experimental design included the tribological properties of biomimetic samples with different texture densities under different load conditions of $100 \mathrm{rpm}$, different rotation speeds of $300 \mathrm{~N}$ load conditions, and characteristic experiments under $100 \mathrm{~N}$ load conditions of $300 \mathrm{rpm}$. The diameter of the unit was $1200 \mu \mathrm{m}$ and the $75 \mathrm{mg}$ of Polyalkylene Glycol (PAG) lubricant was applied uniformly to the surface of the samples. The performance indexes of PAG lubricants are shown in Table 5. The $45 \mathrm{~min}$ tests were designed to study the aggravated running-in caused by material bumping. With such a minute amount of lubricant at the interface, the operating regime was closer to the boundary than the mixed regime and thus considered starved. The diameter and thickness of the GCr15 steel mating discs were 70 and $15 \mathrm{~mm}$, respectively, with a surface roughness of $0.63 \mu \mathrm{m}$. The rotation radius of the specimen was $20 \mathrm{~mm}$. Before the sliding wear test, the mating disc was cleaned in acetone using an ultrasonic vibrator for $10 \mathrm{~min}$. The samples were thoroughly cleaned with acetone in an ultrasonic vibrator for $10 \mathrm{~min}$ before and after the wear test, then dried and weighed with an electronic balance with precision of about $0.01 \mathrm{mg}$. The friction coefficient was acquired directly using the MG-2000 frictiograph, the wear-weight losses were measured by electronic balance with an accuracy of $0.1 \mathrm{mg}$, and the worn surfaces of the samples were observed by SEM. To assess the effect of the textures, the smooth sample was tested for comparison. 


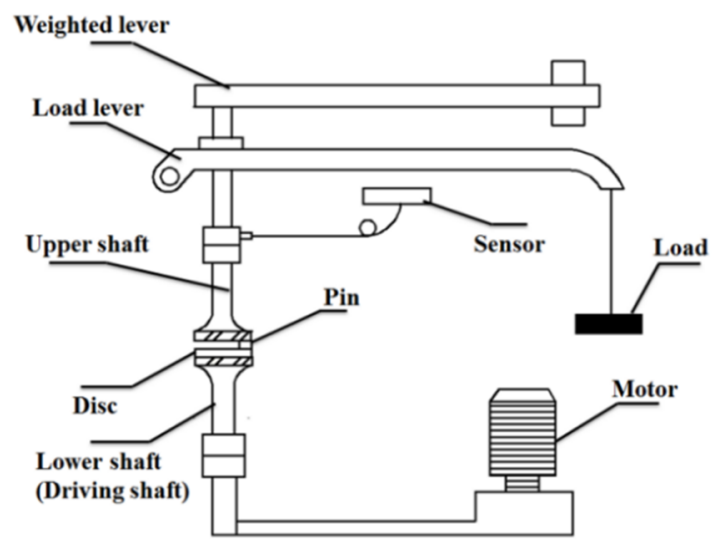

Figure 3. Schematic diagram of the friction and wear tester.

Table 4. The frictional experiment arrangement of smooth and different texture densities under different loads and different speeds.

\begin{tabular}{ccc}
\hline Number & Rotating Speed (rpm) & Load (N) \\
\hline 1 & 100 & 50 \\
2 & 100 & 100 \\
3 & 100 & 200 \\
4 & 100 & 300 \\
5 & 50 & 300 \\
6 & 200 & 300 \\
7 & 300 & 300 \\
8 & 300 & 100 \\
\hline
\end{tabular}

Table 5. The performance index of Polyalkylene Glycol (PAG) lubricating oil.

\begin{tabular}{cccccc}
\hline $\begin{array}{c}\text { Viscosity } \\
\text { Index }\end{array}$ & $\begin{array}{c}\text { Saybolt Universal } \\
\text { Viscosity (SUS) } \\
\mathbf{1 0 0}{ }^{\circ} \mathbf{F}\end{array}$ & $\begin{array}{c}\text { Viscosity } \\
\mathbf{4 0}{ }^{\circ} \mathbf{C}\end{array}$ & $\begin{array}{c}\text { Viscosity } \\
\mathbf{1 0 0}{ }^{\circ} \mathbf{C}\end{array}$ & $\begin{array}{c}\text { Flash Point } \\
{ }^{\circ} \mathbf{C}\end{array}$ & $\begin{array}{c}\text { Pour Point } \\
{ }^{\circ} \mathbf{C}\end{array}$ \\
\hline 197 & 170 & 33 & 7.45 & 232 & -42 \\
\hline
\end{tabular}

\section{Results}

\subsection{Microstructure and Microhardness of the Bionic Unit}

Figure 4 presents that the microstructures of the biomimetic surfaces were different from those of the smooth surfaces. The biomimetic unit included three different zones: the melting zone, where materials were melted completely; the phase-transition zone, where the transformation took place; and the substrate, where no changes occurred (Figure 4a). As shown in the XRD pattern (Figure 5), the substrate was composed of coarse pearlite ferrite. As melting and solidification occur within an extremely short interaction time and are confined only to the top surface, the substrate can act as an infinite heat-sink without any noticeable change in the microstructure [31]. The microstructure of the melting zone (Figure $4 \mathrm{~b}$ ) was mainly composed of martensite and a small amount of retained austenite. In the phase-transition zone (Figure 4c), where the heating temperature was lower than in the melting zone, part of the austenite could not be transformed into martensite and instead formed a mixed-type martensite and a large amount of retained austenite, such that the crystal size of the heat-affected zone was larger than that of the melting zone [32,33]. It can be seen by SEM that the microstructure of the melting zone and phase-transition zone was much more refined and homogeneous than that of the substrate grains. 

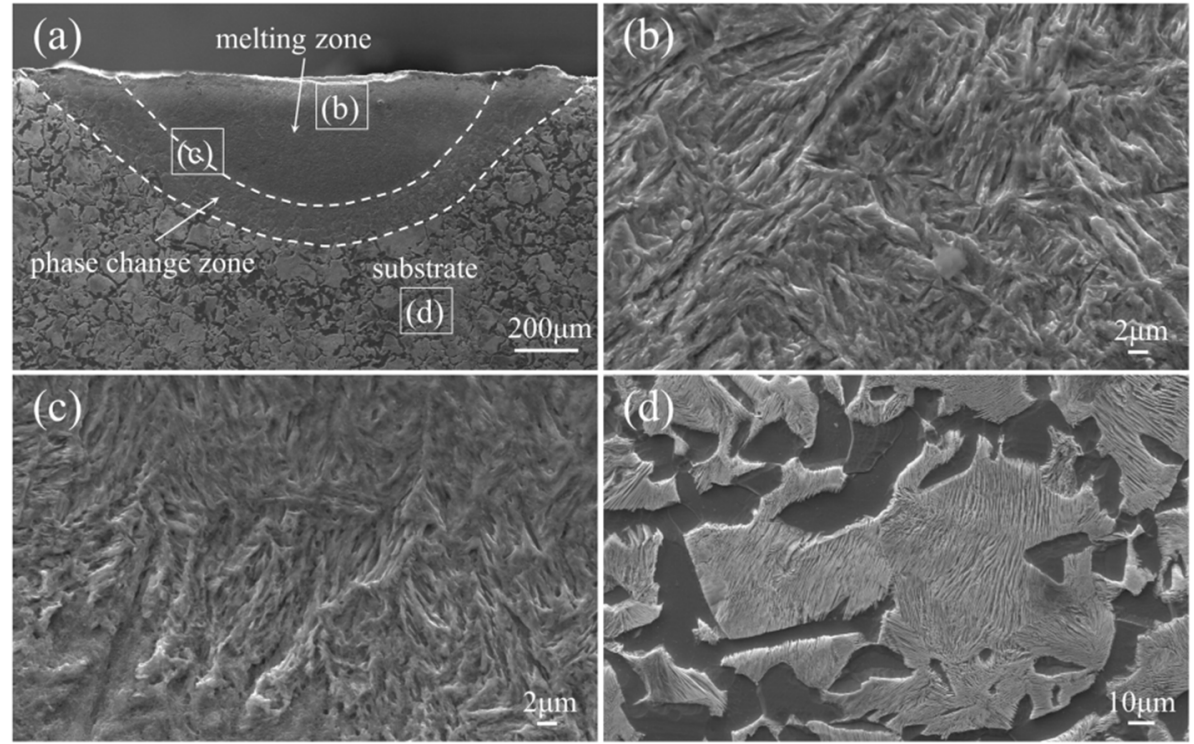

Figure 4. Microstructures of the cross-section in a bionic unit: (a) cross-section of the biomimetic unit, (b) melting zone, (c) phase-transition zone, and (d) substrate.

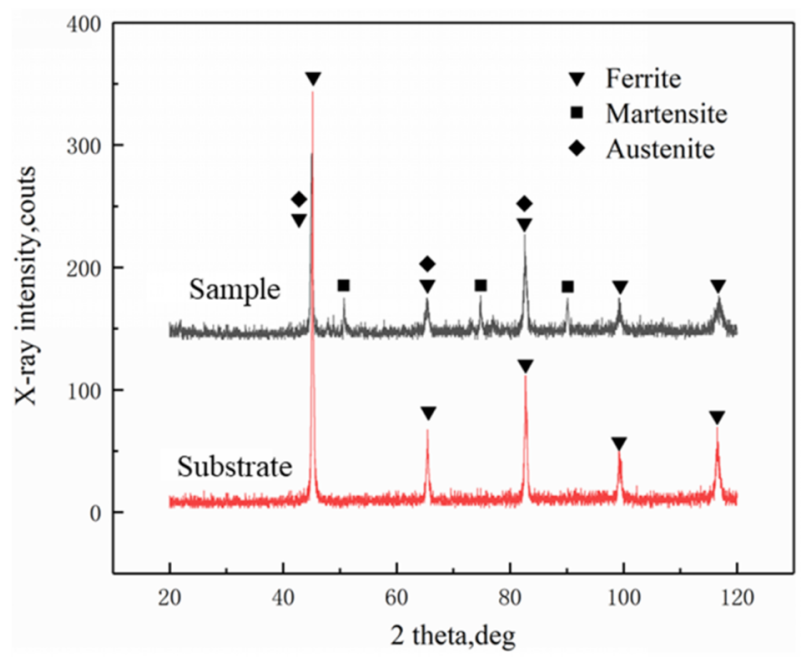

Figure 5. X-ray diffraction (XRD) pattern of the laser-treated medium-carbon steel surface and the untreated surface.

The hardness distribution curve along the depth of the cross-section of the bionic unit is shown in Figure 6. Due to the inhomogeneity of the microstructure, there were great differences in the microhardness between the biomimetic unit and the substrate. The hardest region of the micro-dimple was the heat-affected zone, which possessed a uniform martensitic microstructure after rapid melting and solidification. Due to the finer structure, solid solution, and dislocation, the hardness value of the heat-affected zone increased to $786 \mathrm{Hv}$, which was higher than that of the melted zone $(735 \mathrm{Hv})$. 


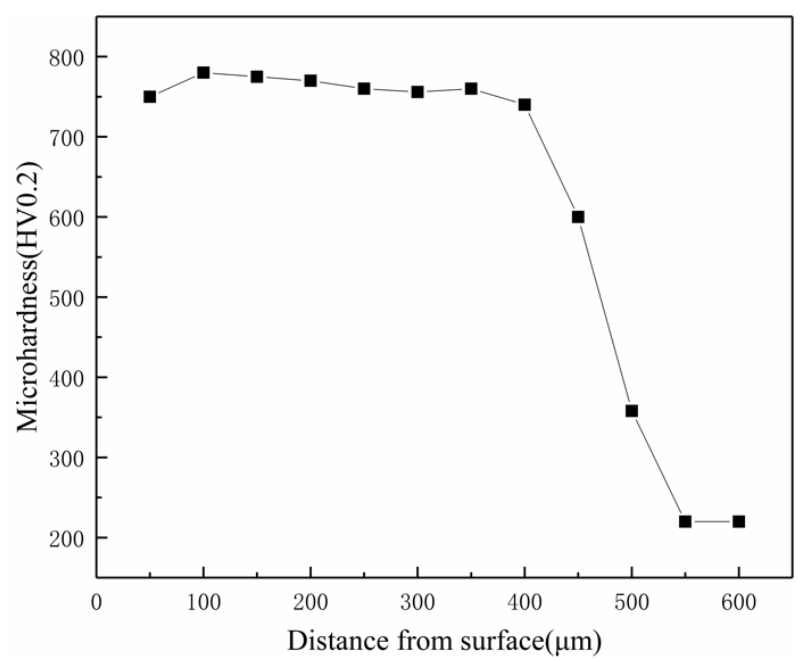

Figure 6. Microhardness curve of the cross-section in a bionic unit.

\subsection{Wear Behavior}

\subsubsection{Friction Properties of Specimens with Different Dimples Densities}

Figure 7 describes the friction properties of different bionic specimens under the conditions of $100 \mathrm{~N}$ and $300 \mathrm{rpm}$. Over time, the friction pair gradually transformed from oil lubrication to starved lubrication, with the friction coefficient of the friction pair remaining at a relatively stable value for a certain period of time, but increasing gradually with the loss of lubricant. In this experiment, bionic samples with texture densities of $4 \%, 8 \%$, and $30 \%$ enter the stage of oil-deficient lubrication in a relatively short time, and the friction coefficient increases at a relatively high rate. Among all of the specimens, the friction coefficient of specimens with $13 \%$ and $18 \%$ texture densities were more stable than other bionic specimens and the smooth specimen.

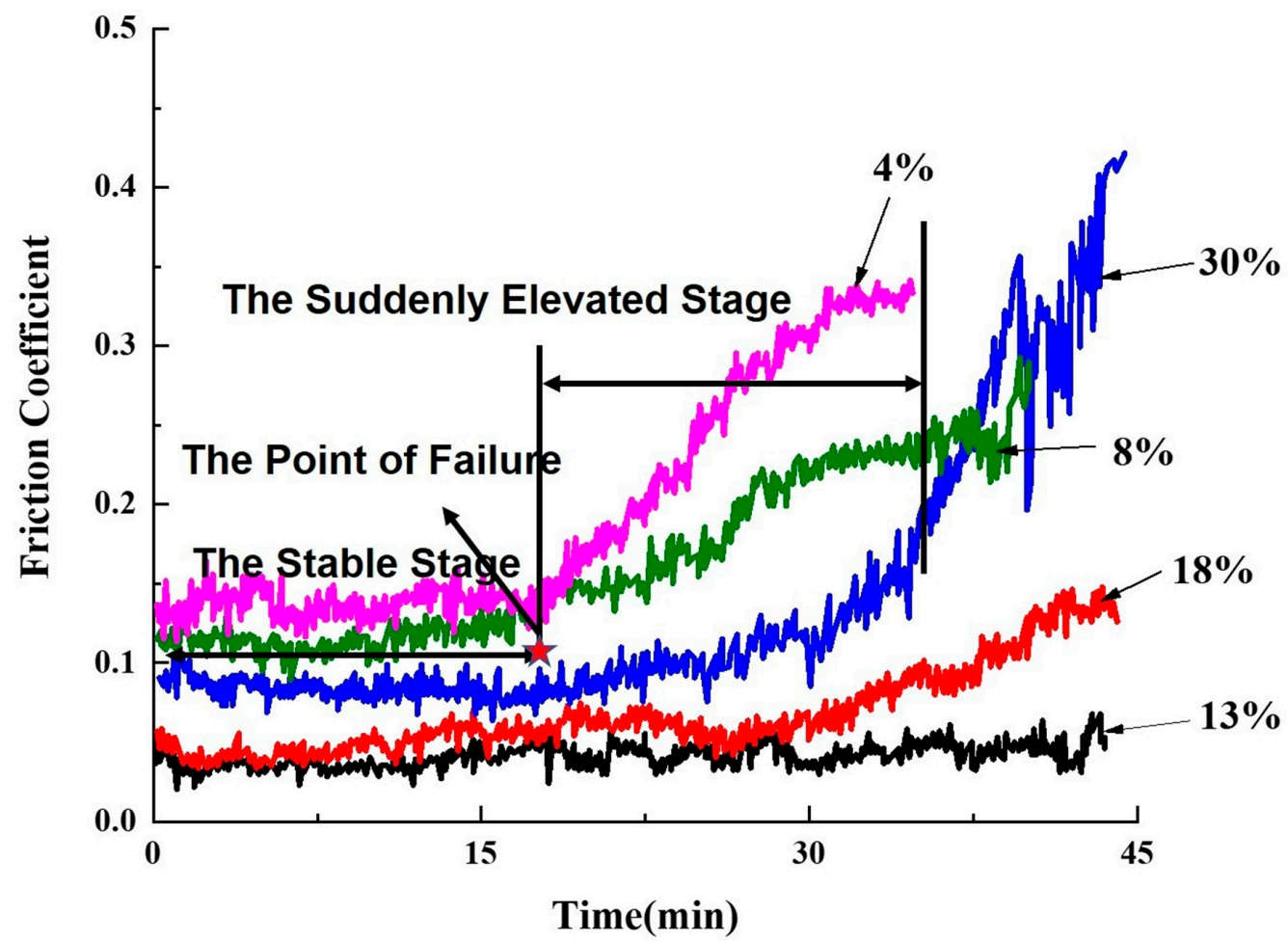

Figure 7. The friction coefficient curves of the biomimetic specimens. 
Compared with the smooth specimen, the bionic specimens possessed lower friction coefficients, and the friction coefficient of the specimen with a $13 \%$ texture density had the lowest value and the longest stable period. The results indicate that the biomimetic units significantly improved the friction performance of the specimen surfaces and that there was an optimal texture density for the friction and wear properties. If the texture density was too low or too high, the friction coefficient increased, and the failure time was shortened. A low texture density meant a limited number of micro-dimples, which limited the reserve lubricant capacity and led to a greater friction coefficient and a shorter stable period. When the texture density was too high, relative movement between the friction pair led to greater lateral resistance, thereby increasing the friction coefficient values. Furthermore, too-high texture density led to high contact pressure, resulting in a large deformation and the appearance of small gaps between the contact surfaces, making the oil film thinner and more readily able to escape from the interface.

\subsubsection{Influence of Load in the Running-in Process on the Tests}

Figure 8a shows the relationship between the tribological performance and different loads in the stable stage. Compared with the smooth specimens, the biomimetic specimens had low friction coefficient values. Among all of the specimens, the friction coefficient of the specimen with a 13\% texture density was the most stable, and it had the lowest values under different load conditions. When the texture density was too low, the ability to reserve oil and debris was limited, and the lubricant of the specimen surfaces was readily consumed, resulting in interface rupture and an increased friction coefficient. When the texture density was too high, the high area density was accompanied by greater frictional resistance, and it was easier to pierce the disc surface and damage the balance of the working system, which led to a greater friction coefficient. When the load was $50 \mathrm{~N}$ or $100 \mathrm{~N}$, the friction coefficient of each specimen decreased with the increase of the load. As the pre-coated oil viscosity of the specimen surfaces increased with the increase of the load, the real sliding friction area of the interface increased, resulting in a reduced friction coefficient. When the load was $200 \mathrm{~N}$ or $300 \mathrm{~N}$, the friction coefficient rose as the load increased. As the load increased, the biomimetic units of the specimen surfaces produced plastic deformation and prevented the oil from entering the interface.
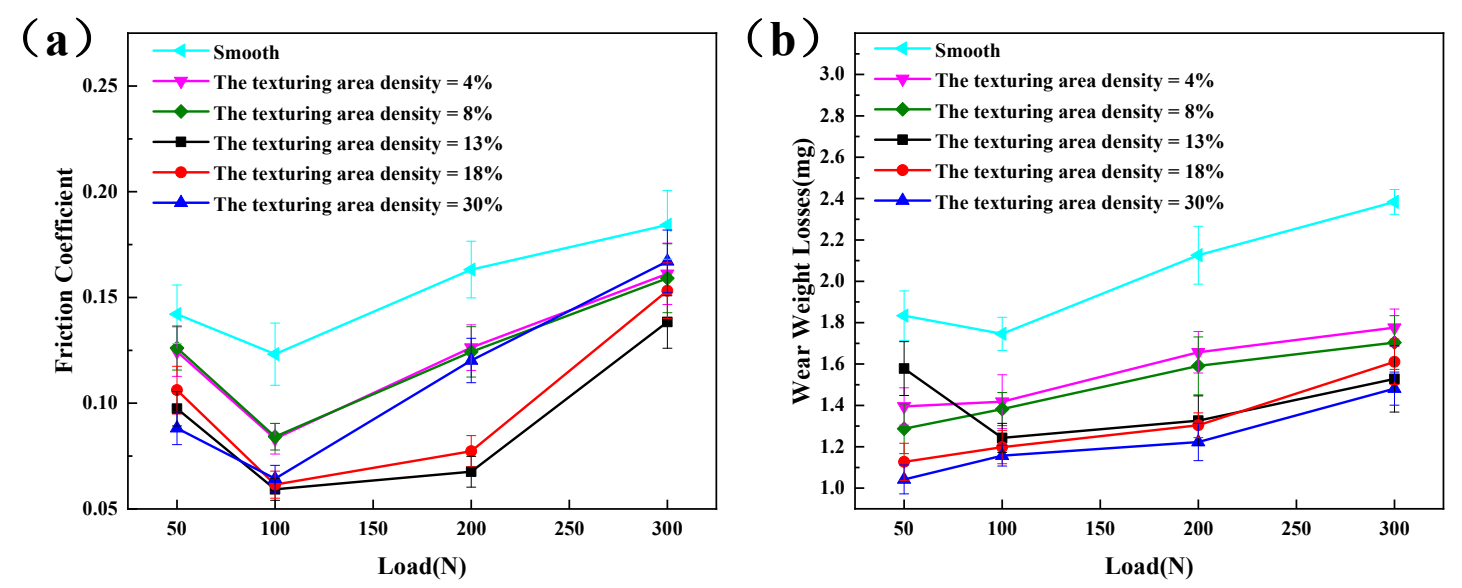

Figure 8. Effect of different texture densities on the friction coefficient and the wear-weight loss at a constant speed of $100 \mathrm{rpm}$ : (a) friction coefficient, and (b) wear-weight losses.

Figure $8 \mathrm{~b}$ depicts the wear-weight losses of the specimens under different loads in the stable stage. As the load increased, the wear-weight losses also increased. The wear-weight losses of the biomimetic specimens were less than that of the smooth specimen. The specimen with a $30 \%$ texture density had the lowest wear-weight loss among all of the biomimetic specimens, which was almost half that of the smooth specimen. The surfaces had similar biological properties to that of rigid-soft coupling through the laser treatment: the biomimetic units with high hardness could resist the wear, and the 
soft substrate could absorb the far-field stress. All these factors increased load carrying capacity and improved wear resistance.

\subsubsection{Influence of Rotating Speed in the Running-in Process on the Tests}

The diagram of the friction coefficient-rotating speed of the biomimetic specimens and the smooth specimen in the stable stage are displayed in Figure 9a. Compared with that of the smooth sample, the friction coefficients of the biomimetic samples were smaller. Among all the biomimetic samples, the specimen with a $13 \%$ texture density had the smallest friction coefficient. The friction coefficients of all the biomimetic specimens decreased as the rotating speed increased, mainly because as the rotating speed increased, more lubricant was brought into the interface, the spoiler effect of the contact surface was strengthened, and it became easier to produce an oil film, which decreased the friction coefficient. Given the same total number of rotations, the faster rotating speed shortened the run-in period, which decreased the wear-weight losses of the run-in period. Moreover, the faster rotating speed helped to discharge the debris rapidly and made the interface temperature increase so that the oxide film formed more readily.
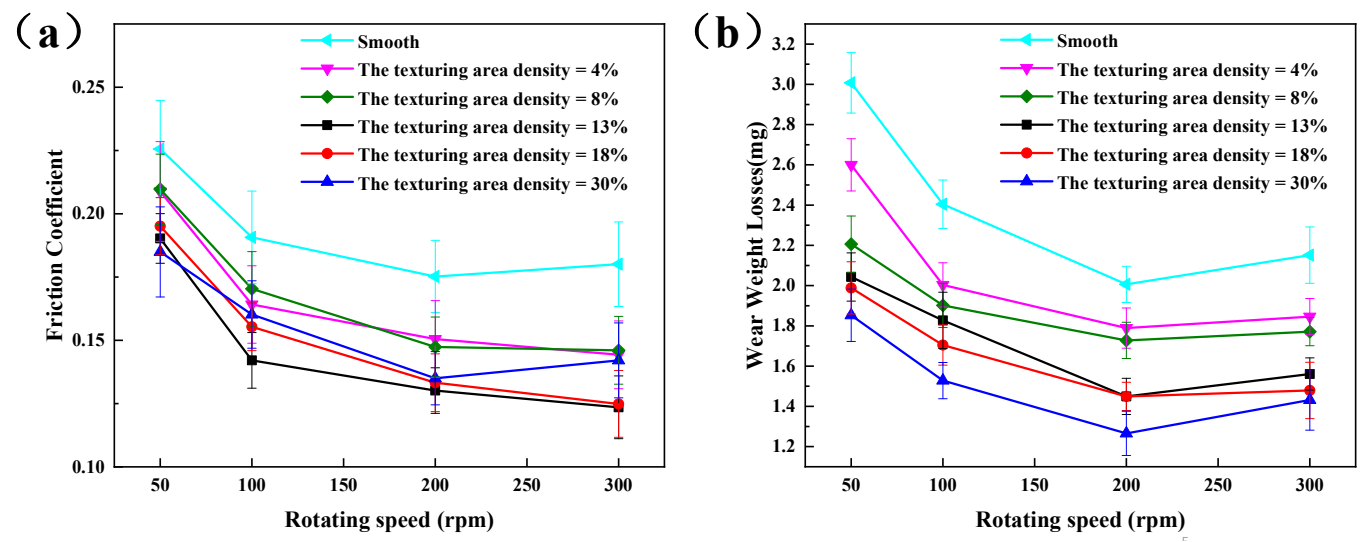

Figure 9. Effect of different texture densities on friction coefficient and wear-weight loss under the constant 300N load: (a) friction coefficient, and (b) wear-weight losses.

Figure $9 \mathrm{~b}$ shows the wear-weight losses of the biomimetic specimens with different rotating speeds in the stable stage. Under the constant load of $300 \mathrm{~N}$, as the rotating speed increased, the wear-weight losses decreased. The specimen with a 30\% texture density exhibited the lowest wear-weight loss among all the biomimetic specimens. When the rotating speed changed, the friction coefficient was stable, and the wear-weight loss was performed steadily, indicating that the biomimetic specimens also had good wear resistance under changing rotating speeds.

\section{Discussion}

\subsection{Tribological Mechanism Analysis}

Obviously, the tribological performance of the bionic specimens is mainly determined by the microstructure of the bionic unit and the base material. Under starved lubrication, because of the limited lubricant storage capacity of the smooth surface, the friction pair has direct contact, which results in serious wear. The SEM images of wear scars for samples with different texture densities after a $45 \mathrm{~min}$ sliding test performed under conditions of $100 \mathrm{~N}$ and $300 \mathrm{rpm}$ are shown in Figure 10. Figure 10a shows that the surface of the smooth specimen was almost worn out. Grooves and frictional particles could be observed, indicating that both abrasive wear and adhesive wear occurred during the test. 


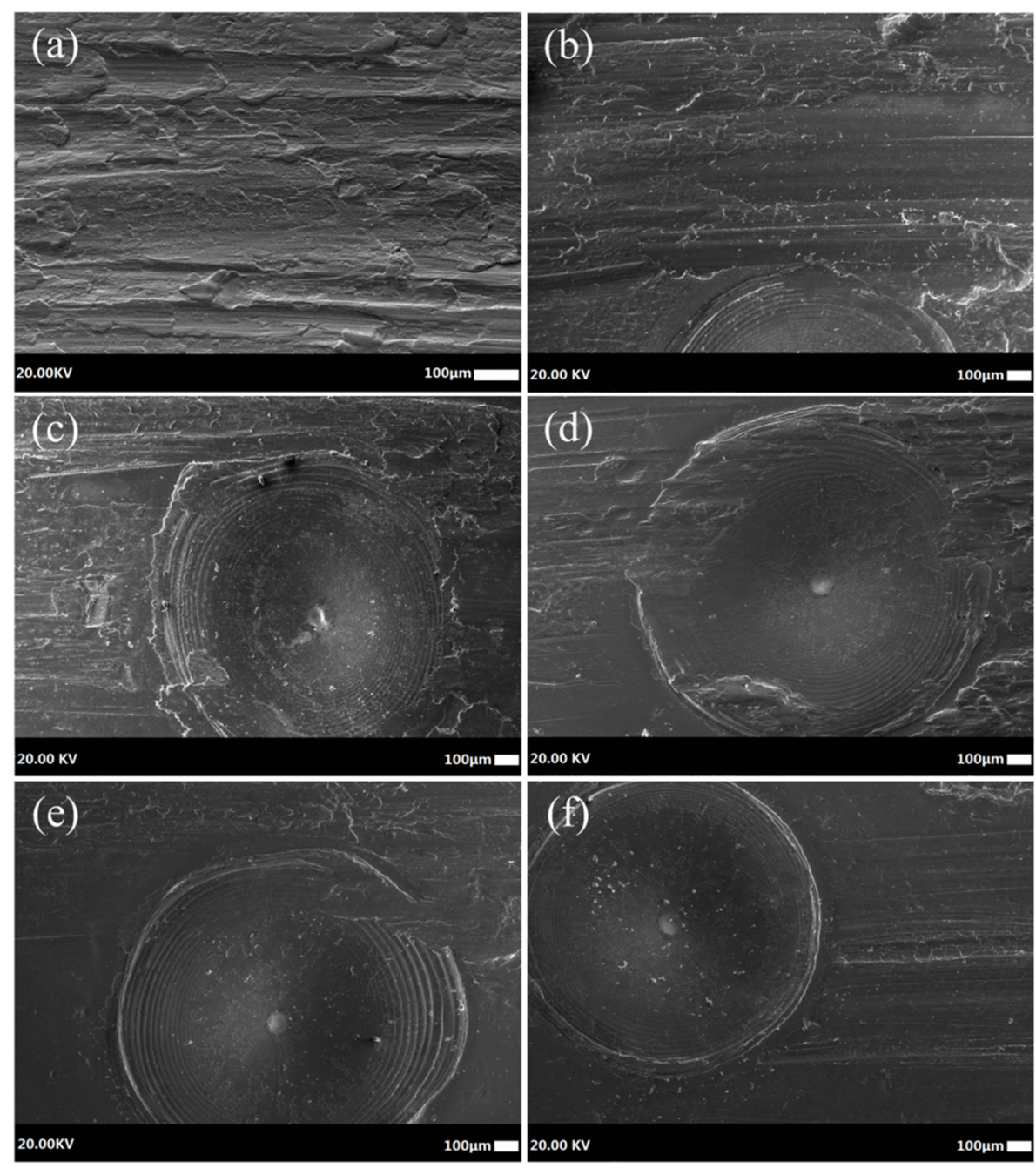

Figure 10. SEM images of wear scars for samples with different texture densities after a 45 min sliding test was performed under conditions of $100 \mathrm{~N}$ and $300 \mathrm{rpm}$ : (a) smooth, (b) texture density $=4 \%$, (c) texture density $=8 \%$, (d) texture density $=13 \%$, (e) texture density $=18 \%$, and (f) texture density $=30 \%$.

There is no doubt that the bionic units are beneficial to friction performance. There are two main theoretical focuses on the lubrication effect of surface texturing. One is the hydrodynamic effect, which occurs mainly in the full- or mixed-lubrication conditions. The other is secondary lubrication, which works in cases of mixed- or starved-lubrication conditions. Few studies have focused on the influence of bionic units with different unit densities on friction and wear. However, with different texture densities friction and wear vary greatly under starved lubrication.

As shown in Figure 10b,c, it can be observed that the bionic units with a $4 \%$ and $8 \%$ texture density have multiple, sometimes deep, scratches on the surface under conditions of $100 \mathrm{~N}$ and $300 \mathrm{rpm}$, thus it can be inferred that the wear-resisting properties of the specimen surface quickly disappeared. Figure $10 \mathrm{~d}$ shows the worn surfaces of the $13 \%$ texture density specimen. Relatively shallow traces of wear were observed on this surface, and most of the original surface material could still be observed clearly, indicating that its biomimetic structure might have had a relatively good effect over time. In the wear rate graph, the curves with different texture densities are obviously different. No significant wear marks are observed on the surfaces in Figure 10e,f, which indicates that the surfaces effectively captured and stored lubricating oil and abrasive particles, thereby significantly preventing abrasive and adhesive wear. In summary, biomimetic structures can have a local strengthening effect, thereby increasing surface hardness. This proves that as the texture density increases, the wear resistance improves. It should be noted that as the spacing of the bionic units decreases, the surface roughness of the friction 
pair increases, causing the friction coefficient to fluctuate greatly, which explains why the friction coefficient of the biomimetic surface increases when the density of the texture exceeds a certain value.

\subsection{Equivalent Stress Distribution of Specimens with Different Texture Densities}

In the friction process, the magnitude and distribution of the contact surface stress greatly affects the wear of the friction surface $[34,35]$. In this section, we explain why the bionic surface can improve the tribological properties by the establishment of finite element models. The simplified model, shown in Figure 11, consists of two parts: a fixed block and a test block above it. In the simulation experiment, the contact surface of the test block was the "master surface" and contact surface of the fixed block was the "slave surface". The material parameters are shown in Table 6. In the experiment, pressure of $15 \mathrm{MPa}$ was uniformly distributed on the test block, then the displacement was applied in the horizontal direction of the test block, and the oil lubrication state was simulated by setting the friction coefficient to 0.15 . The grid number of the smooth sample is 150,000 , and the grid numbers of the biomimetic plates with $4 \%, 8 \%, 13 \%, 18 \%$, and $30 \%$ texture densities are 210,649, 236,331, 248,056, 285,959 , and 302,653 , respectively.

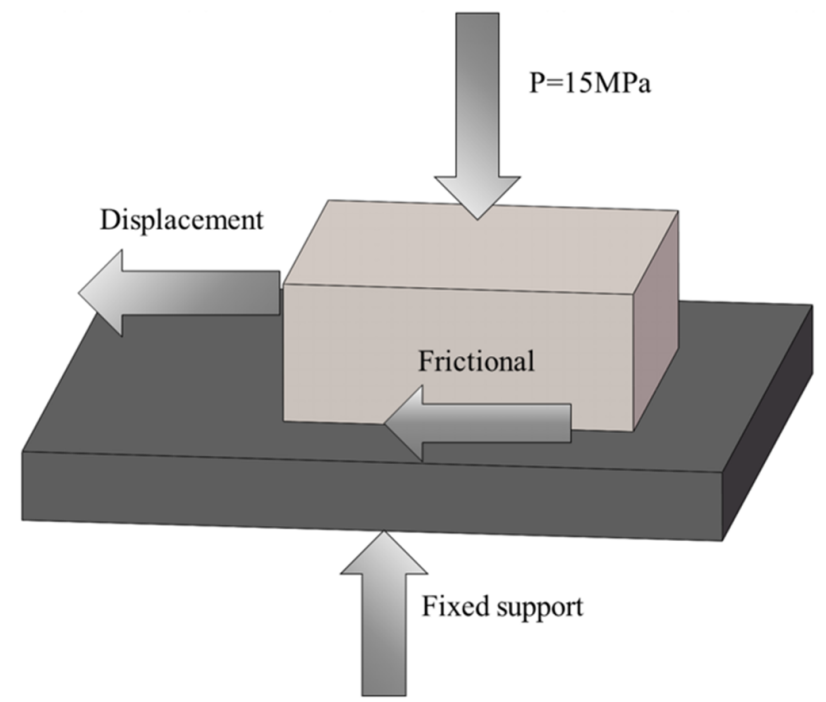

Figure 11. The schematic of the physical model.

Table 6. The performance parameters of medium-carbon steel and GCr15.

\begin{tabular}{ccccccc}
\hline Material & $\begin{array}{c}\text { Density } \\
\left(\mathbf{g} / \mathbf{c m}^{\mathbf{3}} \mathbf{)}\right.\end{array}$ & $\begin{array}{c}\text { Elasticity } \\
\mathbf{( G P a )}\end{array}$ & $\begin{array}{c}\text { Tensile } \\
\text { Strength } \mathbf{( M P a )}\end{array}$ & $\begin{array}{c}\text { Yield Strength } \\
\mathbf{( M P a )}\end{array}$ & $\begin{array}{c}\text { Poisson's } \\
\text { Ratio }\end{array}$ & $\begin{array}{c}\text { Young's Modulus } \\
\mathbf{( G P a )}\end{array}$ \\
\hline $\begin{array}{c}\text { Medium-carbon } \\
\text { steel }\end{array}$ & 7.84 & 207 & 650 & 400 & 0.278 & 206 \\
GCr15 & 7.81 & 210 & 861.3 & 518.4 & 0.300 & 210 \\
\hline
\end{tabular}

The equivalent stresses (von Mises stress) are important in the causation of wear [36]. In this experiment, a numerical study was carried out by Workbench to analyze the influence of three main stresses on plastic damage. The expression can be written as follows:

$$
\sigma_{\mathrm{s}}=\sqrt{\frac{1}{2}\left[\left(\sigma_{1}-\sigma_{2}\right)^{2}+\left(\sigma_{2}-\sigma_{3}\right)^{2}+\left(\sigma_{3}-\sigma_{1}\right)^{2}\right]}
$$

where $\sigma_{\mathrm{s}}$ is the von Mises stress, and $\sigma_{1}, \sigma_{2}$, and $\sigma_{3}$ are the first, second, and third main stress, respectively.

As shown in Figure 12, the large stress was mainly distributed around the edge of the sample surface. Compared with the smooth sample, the large stress region of the biomimetic sample was 
smaller than that of the smooth sample, indicating that the bionic unit could effectively prevent excessive stress concentration. The stress values of most areas of the biomimetic specimen surfaces were smaller than those of the smooth specimen, indicating that the bionic unit could promote uniform stress distribution. The densities of different textured regions had different stress distribution images. When the texture density was between $4 \%$ and $30 \%$, the maximum equivalent stress value decreased first and then increased, proving that the equivalent pressure distribution was affected by the texture area density. The $13 \%$ texture density specimen had the lowest equivalent stress value, which was consistent with the friction experiment results. This finding also verifies the relationship between the tribological properties and stress distribution. In addition, the maximum stress value of the smooth specimen was smaller than that of the bionic specimen, as the contact area of the smooth specimen was larger when the same pressure was applied, resulting in a smaller maximum stress value.
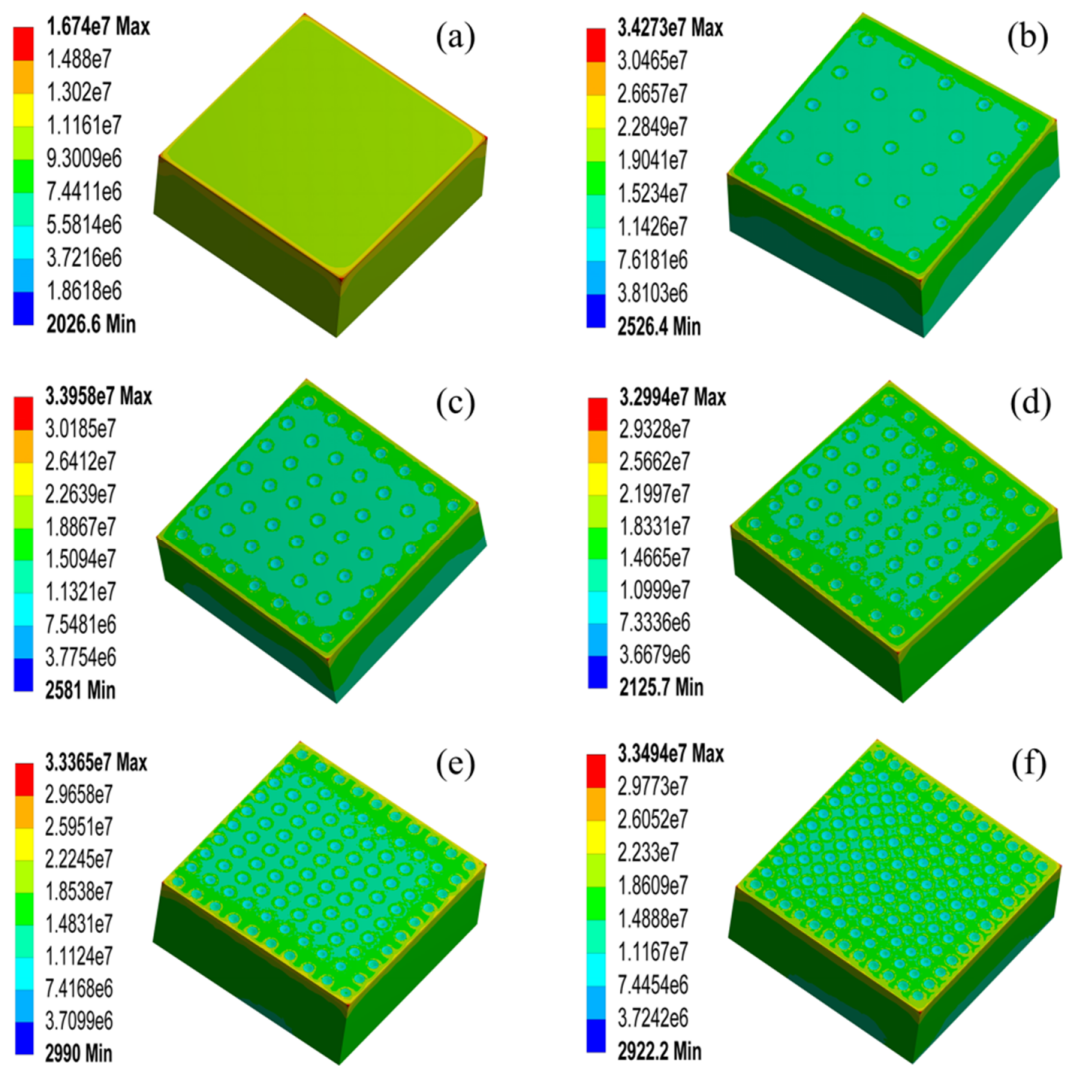

Figure 12. The equivalent stress distributions of the specimens with different texture densities: (a) smooth, (b) texture density $=4 \%$, (c) texture density $=8 \%$, (d) texture density $=13 \%$, (e) texture density $=18 \%$, and (f) texture density $=30 \%$.

\subsection{Further Discussion}

Figure 13a,b show the models of the bionic units capturing and retaining the lubricants, as well as reducing abrasive and adhesive wear. The lubricant trapped in the region of the biomimetic units can be considered a secondary source of lubricant, as it is drawn up by the relative movement to permeate the surface and to reduce the friction and improve wear resistance $[37,38]$. Compared with the base material, the microhardness of the bionic unit was higher, and the abrasive particles had more difficulty invading the material surface, so the movement mode changed from sliding to rolling (Figure 13c). Due to the biomimetic units, when the abrasive particles slid and rolled on the surfaces and produced grooves, the units could prevent the moving path of the particles or make the movement path segregated, thereby effectively stopping the expansion of the cracks and grooves. The bionic units obtained by laser processing have a special microstructure and higher hardness zones when compared 
with the smooth surfaces, which improves the overall resistance to deformation. When the coupling area density increased, the area of hardening produced by the laser thermal effect was amplified, which provided strong support for the deformation resistance and improved wear resistance.
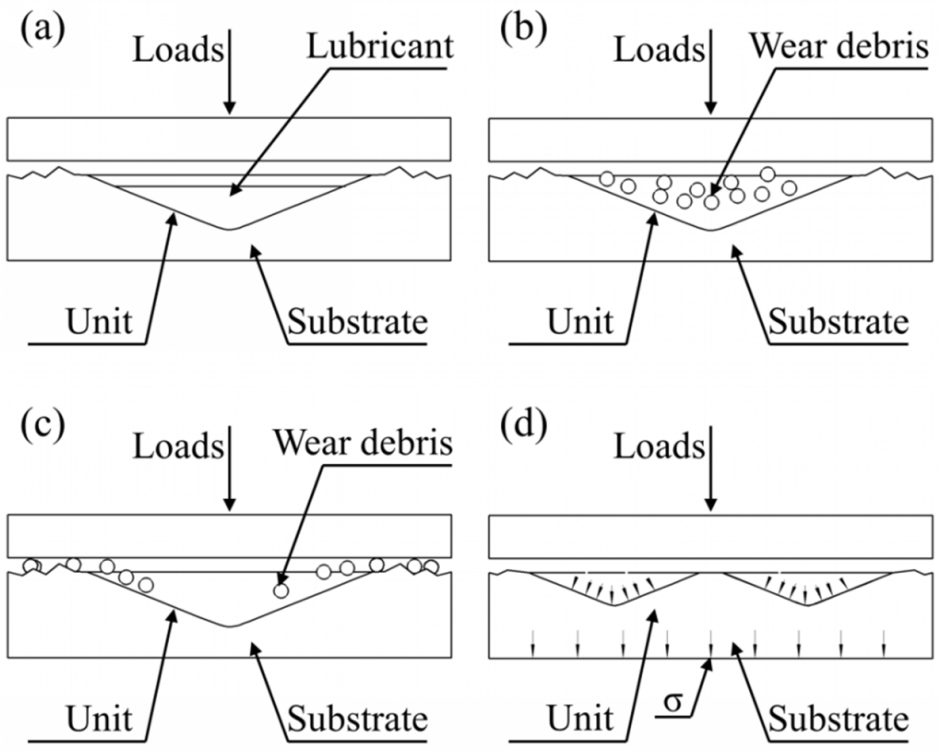

Figure 13. Bionic unit function diagram: (a) storing lubricant, (b) trapping abrasive particles, (c) movement of the abrasive particles from sliding to rolling, and (d) uniform stress distribution effect.

Although the high area density of the coupling unit contributed to the stability of the friction coefficient and wear-weight loss, many factors limited the increase of the area density. As shown in Figure 7 , the specimens with texture densities of $13 \%$ and $18 \%$ performed better than the specimen with an area density of $30 \%$. Moreover, with the increase of grinding time, the friction coefficient of samples with texture densities of $13 \%$ and $18 \%$ remained low and stable. As more roughness greatly restricted the secondary lubrication effect and accelerated the wear of the machine parts, the high coupling density resulted in severe roughness on the surface and led to a high friction coefficient value. As shown in Figure 13d, redistribution of the stress between friction pairs helped to prevent local severe frictional wear. When the area density of the units increased appropriately, the range and standard deviation of the equivalent stress became smaller, meaning that the equivalent stress distribution became uniform. However, as the area density continued to increase, the maximum value, range, and standard deviation of the equivalent stress increased, meaning there was a most suitable area density to minimize the equivalent stress.

\section{Conclusions}

Five densities of textures were fabricated onto the surfaces of medium-carbon steel by using laser technology, and friction tests were carried out on a steel CGr15 disc under starved-lubrication conditions. The main conclusions from this research are as follows:

- Among all the specimens, biomimetic specimens with various coupling area densities showed better performance than the smooth specimen, because they benefited from high hardness and varying subsurface structures.

- Among the biomimetic surfaces, the friction coefficient of the specimen with an area density of $13 \%$ was lower than those with low area density specimens $(4 \%, 9 \%)$ and high area density specimens $(18 \%, 30 \%)$. The wear-weight loss of the $30 \%$ specimen was less than that of the others.

- As the load was increased, the friction coefficient values of the biomimetic specimens first decreased (under small loads between 50 and $100 \mathrm{~N}$ ) and then increased (under large loads between 200 and $300 \mathrm{~N}$ ). As the rotating speed increased, the friction coefficient values of the 
biomimetic specimens gradually decreased. Regardless of the load and rotating speed conditions, the biomimetic specimens displayed excellent wear resistance.

Author Contributions: F.Z. and Y.L.; Literature, F.S.; data collection, X.L.; figures, Z.Y., Y.G., and Z.W.; formal analysis, L.L.; writing, Z.Z.; study design. All authors have read and agreed to the published version of the manuscript.

Funding: This research was funded by the National Key R\&D Program of China (No. 2018YFB1105100), the National Natural Science Foundation of China (No. 51975246), the Ascl-zytsxm (202013), Science and Technology Project of Jilin Education Department (JJKH20200958KJ), the Program for JLU Science and Technology Innovative Research Team (No. 2019TD-34) and the Advanced Manufacturing Project of Provincial School Construction of Jilin Province (No. SXGJSF2017-2), the National Natural Science Foundation of China (No. U1601203), and Postdoctoral Science Foundation of China (No. 801161050414).

Conflicts of Interest: The authors declare no conflict of interest.

\section{References}

1. Kim, H.-J.; Yoo, S.-S.; Kim, D.-E. Nano-scale wear: A review. Int. J. Precis. Eng. Manuf. 2012, 13, $1709-1718$. [CrossRef]

2. Zhang, J.; Meng, Y. Boundary lubrication by adsorption film. Friction 2015, 3, 115-147. [CrossRef]

3. Anand, M.; Hadfield, M.; Viesca, J.L.; Thomas, B.; Battez, A.H.; Austen, S. Ionic liquids as tribological performance improving additive for in-service and used fully-formulated diesel engine lubricants. Wear 2015, 334, 67-74. [CrossRef]

4. Akram, M.W.; Polychronopoulou, K.; Polycarpou, A.A. Tribological performance comparing different refrigerant-lubricant systems: The case of environmentally friendly HFO-1234yf refrigerant. Tribol. Int. 2014, 78, 176-186. [CrossRef]

5. Zhang, P.; Lu, W.; Liu, X.; Zhai, W.; Zhou, M.; Jiang, X. A comparative study on torsional fretting and torsional sliding wear of $\mathrm{CuNiAl}$ under different lubricated conditions. Tribol. Int. 2018, 117, 78-86. [CrossRef]

6. Dong, C.L.; Yuan, C.Q.; Bai, X.Q.; Yang, Y.; Yan, X.P. Study on wear behaviours for NBR/stainless steel under sand water-lubricated conditions. Wear 2015, 332, 1012-1020. [CrossRef]

7. Gnilitskyi, I.; Rotundo, F.; Martini, C.; Pavlov, I.; Ilday, S.; Vovk, E.; Ilday, F.O.; Orazi, L. Nano patterning of AISI 316L stainless steel with nonlinear laser lithography: Sliding under dry and oil-lubricated conditions. Tribol. Int. 2016, 99, 67-76. [CrossRef]

8. Sui, Q.; Zhou, H.; Yang, L.; Zhang, H.; Peng, L.; Zhang, P. Couple of biomimetic surfaces with different morphologies for remanufacturing nonuniform wear rail surface. Opt. Laser Technol. 2018, 99, 333-341. [CrossRef]

9. Sudeep, U.; Tandon, N.; Pandey, R.K. Performance of lubricated rolling/sliding concentrated contacts with surface textures: A review. J. Tribol.-Trans. Asme 2015, 137, 031501. [CrossRef]

10. Liu, D.; Yan, B.; Shen, B.; Liu, L.; Hu, W. Friction behaviors of rough chromium surfaces under starving lubrication conditions. Appl. Surf. Sci. 2018, 427, 857-862. [CrossRef]

11. Wos, S.; Koszela, W.; Pawlus, P.; Drabik, J.; Rogos, E. Effects of surface texturing and kind of lubricant on the coefficient of friction at ambient and elevated temperatures. Tribol. Int. 2018, 117, 174-179. [CrossRef]

12. Vladescu, S.-C.; Ciniero, A.; Tufail, K.; Gangopadhyay, A.; Reddyhoff, T. Looking into a laser textured piston ring-liner contact. Tribol. Int. 2017, 115, 140-153. [CrossRef]

13. Wang, P.F.; Han, Z.; Lu, K. Enhanced tribological performance of a gradient nanostructured interstitial-free steel. Wear 2018, 402, 100-108. [CrossRef]

14. Gu, D.; Hagedorn, Y.-C.; Meiners, W.; Meng, G.; Batista, R.J.S.; Wissenbach, K.; Poprawe, R. Densification behavior, microstructure evolution, and wear performance of selective laser melting processed commercially pure titanium. Acta Mater. 2012, 60, 3849-3860. [CrossRef]

15. Sun, J.; Zhou, Y.; Deng, J.; Zhao, J. Effect of hybrid texture combining micro-pits and micro-grooves on cutting performance of WC/Co-based tools. Int. J. Adv. Manuf. Technol. 2016, 86, 3383-3394. [CrossRef]

16. Han, J.; Fang, L.; Sun, J.; Wang, Y.; Ge, S.; Zhu, H. Hydrodynamic lubrication of surfaces with asymmetric microdimple. Tribol. Trans. 2011, 54,607-615. [CrossRef]

17. Etsion, I. State of the art in laser surface texturing. J. Tribol.-Trans. Asme 2005, 127, 248-253. [CrossRef] 
18. Querlioz, E.; Ville, F.; Lenon, H.; Lubrecht, T. Experimental investigations on the contact fatigue life under starved conditions. Tribol. Int. 2007, 40, 1619-1626. [CrossRef]

19. Meng, F.; Zhou, R.; Davis, T.; Cao, J.; Wang, Q.J.; Hua, D.; Liu, J. Study on effect of dimples on friction of parallel surfaces under different sliding conditions. Appl. Surf. Sci. 2010, 256, 2863-2875. [CrossRef]

20. Wu, Z.; Deng, J.; Xing, Y.; Cheng, H.; Zhao, J. Effect of surface texturing on friction properties of WC/Co cemented carbide. Mater. Des. 2012, 41, 142-149. [CrossRef]

21. Pettersson, U.; Jacobson, S. Tribological texturing of steel surfaces with a novel diamond embossing tool technique. Tribol. Int. 2006, 39, 695-700. [CrossRef]

22. Costa, H.L.; Hutchings, I.M. Hydrodynamic lubrication of textured steel surfaces under reciprocating sliding conditions. Tribol. Int. 2007, 40, 1227-1238. [CrossRef]

23. So, H.; Chen, C.H. Effects of micro-wedges formed between parallel surfaces on mixed lubrication - Part II: Modeling. Tribol. Lett. 2005, 19, 83-91. [CrossRef]

24. Anno, J.N.; Walowit, J.A.; Allen, C.M. Microasperity Lubrication. J. Lubr. Tech 1968, 4, 351. [CrossRef]

25. Anno, J.N.; Walowit, J.A.; Allen, C.M. Load Support and Leakage from Microasperity-Lubricated Face Seals. J. Tribol. 1969, 91, 726-731. [CrossRef]

26. Lo, S.W.; Horng, T.C. Lubricant permeation from micro oil pits under intimate contact condition. J. Tribol. 1999, 121, 633. [CrossRef]

27. Ren, L.; Liang, Y. Preliminary studies on the basic factors of bionics. Sci. China-Technol. Sci. 2014, 57, 520-530. [CrossRef]

28. Povarnitsyn, M.E.; Itina, T.E.; Sentis, M.; Khishchenko, K.V.; Levashov, P.R. Material decomposition mechanisms in femtosecond laser interactions with metals. Phys. Rev. B 2007, 75, 235414. [CrossRef]

29. Povarnitsyn, M.E.; Khishchenko, K.V.; Levashov, P.R. Phase transitions in femtosecond laser ablation. Appl. Surf. Sci. 2009, 255, 5120-5124. [CrossRef]

30. Povarnitsyn, M.E.; Khishchenko, K.V.; Levashov, P.R. Simulation of ultrashort double-pulse laser ablation. Appl. Surf. Sci. 2011, 257, 5168-5171. [CrossRef]

31. Kwok, C.T.; Leong, K.I.; Cheng, F.T.; Man, H.C. Microstructural and corrosion characteristics of laser surface-melted plastics mold steels. Mater. Sci. Eng. Struct. Mater. Prop. Microstruct. Process. 2003, 357, 94-103. [CrossRef]

32. Sivanandham, N.; Rajadurai, A.; Shariff, S.M.; Senthilselvan, J.; Mahalingam, A. Microstructure, mechanical properties and corrosion resistance of laser surface melted EN353 low carbon low alloy steel. Int. J. Surf. Sci. Eng. 2017, 11, 118-132. [CrossRef]

33. Vilar, R.; Colaco, R.; Almeida, A. Laser surface treatment of tool steels. Opt. Quantum Electron. 1995, $27,1273-1289$.

34. Zhang, Z.; Shao, F.; Liang, Y.; Lin, P.; Tong, X.; Ren, L. Wear behavior of medium-carbon steel with biomimetic surface under starved lubricated conditions. J. Mater. Eng. Perform. 2017, 26, 3420-3430. [CrossRef]

35. Liang, Y.; Zhao, Q.; Zhang, Z.; Lin, Z.; Ren, L. Fabrication of bionic composite material using self-propagating high-temperature synthesis in the $\mathrm{Cu}-\mathrm{Ti}-\mathrm{B} 4 \mathrm{C}$ system during steel casting. J. Asian Ceram. Soc. 2013, 1, 339-345. [CrossRef]

36. Han, Z.L.; Wang, J.D.; Chen, D.R. The friction-reduce effect with different depth of concave on the oil-lack lubrication. Lubr. Eng. 2007, 32, 18-20.

37. Krupka, I.; Poliscuk, R.; Hartl, M. Behavior of thin viscous boundary films in lubricated contacts between micro-textured surfaces. Tribol. Int. 2009, 42, 535-541. [CrossRef]

38. Menezes, P.L.; Kishore; Kailas, S.V.; Lovell, M.R. Influence of surface texture and roughness of softer and harder counter materials on friction during sliding. J. Mater. Eng. Perform. 2015, 24, 393-403. [CrossRef]

Publisher's Note: MDPI stays neutral with regard to jurisdictional claims in published maps and institutional affiliations. 